\title{
The Western Australian Clinical Senate as a Model for State-Wide Clinical Engagement
}

\author{
Quinlivan JA ${ }^{1 *}$, Basile T², Gibson $\mathrm{K}^{3}, \mathbf{X u} \mathrm{D}^{4}$ and Croker $\mathrm{N}^{5}$ \\ ${ }^{1}$ Department of Health, University of Notre Dame, Institute for Health Research, Western Australia \\ ${ }^{2}$ Sir Charles Gairdner Hospital, Hospital Avenue, Nedlands \\ ${ }^{3}$ Health Sciences, Curtin University, Western Australia \\ ${ }^{4}$ Department of Health, Musculoskeletal Health Network, Australia \\ ${ }^{5}$ University of Notre Dame, Women and Newborn Health Service, Western Australia
}

\begin{abstract}
International reforms in healthcare have established the principal that embracing clinical engagement can drive healthcare reform and improved safety and quality outcomes. Most attention has focussed on how clinicians might be engaged at a local or hospital level. However, healthcare reforms are usually initiated at a state or national level. Less attention has gone into exploring the key elements of a model to achieve clinical engagement at state or national level. This paper explores the key elements of a successful state-wide clinical engagement model including culture and leadership, membership, decision-making processes and accountability and discusses how the current model of the Western Australian Clinical Senate addresses these elements. This model may be applicable in other jurisdictions and healthcare systems.
\end{abstract}

Keywords: Health care; Health systems; National Health; Patients

\section{Introduction}

Over the past 50 years there have been significant changes in the approach to healthcare management [1]. These changes have altered the way many clinicians are engaged in planning, policy and delivery of services [2,3]. In many cases, clinicians have felt less engagement in decision making and felt a broadening gulf between decision maker and the coal face where healthcare is delivered $[2,3]$.

However, reviews like the Griffith and Mid Staffordshire Reports into management in the UK National Health Service have emphasized that the involvement of doctors, and more recently, all clinicians, is necessary in order to achieve healthcare reforms that are safe, effective and efficient $[4,5]$. When clinician engagement is poor, quality and safety is the first to suffer.

\section{However as Clement quoted}

"The evidence is compelling-engaged physicians, staff, and patients are essential to making positive changes and sustainable improvements in health care organizations. Those achieving better clinical outcomes, positive patient experiences and efficient and effective care or services generally have engaged clinicians and staff. Engaged people feel respected, heard, valued, and empowered to contribute to and influence the delivery of health care services [3]." Achieving clinical engagement at a ward, department or hospital level is different to engagement at a state or national level.

Recognising this, WA Health has a strategic objective to support the delivery of excellent patient-centred care and high quality services through meaningful and sustained clinician engagement. Clinician engagement is defined as workforce partnership shaping the direction of the health service towards achieving patient-centred care and fostering a strong sense of ownership of the health service.

WA health are currently progressing a major health reform with the establishment of new health service boards, with the health department adopting a role of system manager. It is essential in this context for the Clinical Senate of WA to deliver an innovative model of clinical engagement. The aim of this article is to review literature of clinician engagement to guide the implementation of the most suitable model for WA Health.

\section{Culture and leadership}

The first element is culture and leadership. Culture is vital to the success of any organisation. The business dictionary defines culture as "The values and behaviours that contribute to the unique social and psychological environment of an organisation [6]. Organisational culture is the sum total of an organisation's past and current assumptions, experiences, philosophy and values that hold it together, and is expressed in its self-image, inner workings, interactions with the outside world and future expectation."

The leadership set the cultural tone of an organisation. One important element of culture is the ethical framework upon which decision-making occurs. In a healthcare framework, ethics plays an even greater role than in other settings. Whilst personal ethics is an easy concept, the notion of "an ethical organisation" is a harder concept to grasp. Inherently it is more than simply an organisation that follows the law and maintains its rules; it implies that all decision-making and activity must hold at its core a philosophy to act in "the right way".

\section{The tone at the top}

In the Australian Institute of Company Directors' module on the role of boards and the practice of directorship, they emphasize the "tone at the top" [7]. Culture starts with the Chair, extends to the Executive or

*Corresponding author: Julie A Quinlivan, Department of Health, 2nd floor C block, 189 Royal Street, East Perth 6004, Western Australia and Institute for Health Research, University of Notre Dame Australia, Mouat St, Fremantle WA, Australia, Tel: 61892224096; E-mail: ClinicalSenate@health.wa.gov.au

Received August 01, 2016; Accepted August 22, 2016; Published August 31 2016

Citation: Quinlivan JA, Basile T, Gibson K, Xu D, Croker N (2016) The Western Australian Clinical Senate as a Model for State-Wide Clinical Engagement. Health Care: Current Reviews 4: 172. doi: 10.4172/2375-4273.1000172

Copyright: (C) 2016 Quinlivan JA, et al. This is an open-access article distributed under the terms of the Creative Commons Attribution License, which permits unrestricted use, distribution, and reproduction in any medium, provided the original author and source are credited. 
Board, and then spreads through the membership and is reflected in the mission and outcomes of the organisation.

In a state-wide clinical engagement body (such as the Clinical Senate of WA), the chair and executive are therefore important for setting both the culture and ethical framework. Who these clinicians are and how they are appointed are therefore important steps in establishing the tone of the organisation.

Across Australia there are different strategies used to harness clinician engagement. Three options for appointments to leadership roles were identified. The first is a Ministerial or Director General appointed Chair. The advantage of this option is a presumption that such an appointee would have facilitated access to the Minister or Director General, providing two way flow of information to clinicians. However, a disadvantage would be the potential for party politics to influence the clinical engagement agenda.

The second option would be for the membership to nominate a Chair, with a decision ratified by the Minister or Director General of Health. The advantage of this process is the ownership of the role of Chair belongs with clinicians, but can be managed by a Minister or Director General to ensure a suitable appointee is nominated. A disadvantage is that there is potential for disagreement between the nominated appointee and the Minister or Director General, and this could lead to friction that derailed an engagement agenda.

The third option is an elected model where membership elects a Chair. The advantage of this model is the process is democratic. As with the first option the disadvantage is that party or factional politics might influence outcomes and detract from the clinical engagement agenda.

In Queensland, the Chair is a Ministerial appointment. However, in Western Australia, the Clinical Senate has adopted the second process, wherein the Senate Executive nominate a Chair and the appointment is ratified by the Director General of Health. The recent survey of the current and immediate past members of the WA Clinical Senate found strong support for an independent Chair nominated by the membership but ratified by the Director General or Minister [8]. This process was seen to retain the independence of the Chair, and yet maintain an environment of mutual respect and engagement at the highest levels of decision making.

\section{The executive or board}

In Queensland the executive are appointed by the Chair. In regard to appointment to the executive or board of the Clinical Senate, in Western Australia members are invited to nominate for executive vacancies. If there is competition, a ballot is held and the successful applicant appointed. An alternative model would be a pure appointment system, or at the other end of the scale, a pure and open election system for all appointments. However, both these alternatives risk in the introduction of factionalism and party politics.

\section{Membership}

The wider membership of a Clinical Senate also determines the culture and ethics of the organisation. Our recent survey of Clinical Senators reinforced the principles of membership contained within the Terms of reference $[8,9]$. These principles included:

- Broad clinician membership across all sectors where health care is delivered (by example, primary, secondary, tertiary, public sector, private sector).

- The consumer voice should be included
- All members are apolitical

- All members should be practicing clinicians

- Each member, although selected from predefined sections of health care are to remain non-representational

- Rural representation should reflect the proportion of rural population.

- There should be a range of clinicians from the hierarchies that exist in health

- Must reflect all disciplines including nursing, medical, allied health and aboriginal health and

- Must be reflective of all aspects of clinical care.

\section{A broad membership}

The Clinical Senate membership in Western Australian (WA) includes 80 clinicians and 2 consumers and operates independently from the formal Department of Health structure [9]. The makeup of this team of clinicians and consumers has been a strong contributing factor of its success. Clinical care is delivered by a diverse group of clinicians and as such the makeup of the Clinical Senate recognises diversity of health care delivery in using the following definition of a clinician $[9,10]$. "A clinician refers to any health practitioner working with the Western Australian health system, either as a public and/or private practitioner."

The definition and makeup of the Senate therefore requires a diverse membership reflecting a range of practitioner voice. It is also testament to the fact that health care is delivered in a wide range of structures and locations. The team diversity must also reflect the cultural needs of the population of WA. Team Diversity is the significant uniqueness of each individual on a team. This should not only include the usual diverse selections such as religion, sex, age and race, but also additional unique personality characteristics such as introverts and extroverts, liberals and conservatives, etc. [11].

In reflecting on the definition above each Senator brings uniqueness to the Clinical Senate. This will in part be about who they are and in part where they deliver health care. The challenge and value of appreciating input from a diverse range of clinicians has been summed as:

"The greatest challenge for clinicians in engaging in clinical service planning, policy driving and health reform of all kinds remains balancing the ever increasing demand for engagement with clinical, academic and research commitment. The answer may lie in valuing the perspectives of all clinicians, across all professional backgrounds, regardless of seniority [12]."

Membership diversity includes not only location and type of practice and clinician; it also includes accounting for age and years of clinical experience. Younger clinicians bring a different perspective, one that is not steeped in the confines of tradition. Older clinicians bring experience and knowledge of prior successes and failures.

\section{Respectful partnerships with consumers}

Clinical engagement and consumer experience are equally important factors, so no clinical engagement forum should lack consumer input. This was clearly stated as follows:

"Engagement of staff and patients is underpinned by a common set of values. In the context of the Engaging Leadership model it can be seen that organisations which engage both groups will be open 
and accessible, will emphasise collaboration, will remove barriers to communication, see the work through the eyes of others, take on board their concerns and perspectives and work with their ideas. Each individual will be valued, supported and listened to [2]."

\section{Apolitical}

Over its' lifetime the Clinical Senate has been seen as a forum where the clinical voice can be heard and considered. Many writers on leadership and engagement state that you get neither if you do not build trust. With trust comes respect. This quality must be displayed by all members of the Senate to ensure each participant feels safe to express an opinion and contribute to the discussion. Cultural safety is yet another area requiring insight and at times sensitive management.

Expectancy theory was introduced in 1964 by Victor Vroom, in his book Work and Motivation [13]. It argues that the strength of your motivation to act in a certain way depends on the strength of your expectation that (a) a given level of activity will cause a given outcome and (b) for a high level of activity, this outcome will be attractive.

There is also a relationship between trust and expectation. If we trust this relationship between expectation and outcome, then motivating people should come down to three things:

1. Effort - encouraging the belief that making more effort will improve performance.

2. Performance - encouraging the belief that a high level of performance will bring a good reward.

3. Outcome - making sure that the reward is attractive.

When these variables are high, we expect motivation to be high: The formula is simple. The challenge is creating - and maintaining a strong link between high effort and peak performance. This is what "Expectancy Theory" says [13]. Because it's based on an intuitive, instinctive understanding of motivation, some say that expectancy theory is the most comprehensive explanation of motivation that we have. Our drive must always be centred around improving healthcare services and systems to meet the needs of our population. With effort and engagement the Clinical Senate of Western Australia has demonstrated success.

Senators understand that the outcomes of the Clinical Senate meetings have led to improving patient care, patient outcomes, health system modelling and innovation in health care delivery.

\section{Senator qualities}

As cited in The Kings Fund paper 2012 "Leadership and the engagement for improvement in the NHS,

"Leadership needs to be developed in ways that break down rather than reinforce silos, the unifying vision for every leader should be engaging for improvement's with a clear focus on improving patient care and population health outcomes [1]."

Words and actions like "lead by example, motivate, inspire, mentor, coach, teamwork, vision and achievement" are all tried and tested in the Clinical Senate arena. Members must be prepared to commit to the prereading to open up their thinking, to actively listen to the experts in the room, to share their experiences and enquire from a place of curiosity rather than threat and challenge. Senators need a sense of humility to recognise that they as individuals do not know it all.

Senators need to recognise the best care is that which is integrated and multidisciplinary in the spirit of collaborative practice. As stated in "Clinical Service Integration: a stocktake of the Australian Experience...

"integration is concerned with the processes of bringing organisations and professionals together, with the aim of improving outcomes for patients and service users through the delivery of integrated care. This could also be seen as collaborative practice, no doubt a pre-condition for integrating care [14]."

\section{Non representational}

In recognising health care is delivered in many locations, including metropolitan, rural and remote, both public and private, from primary to tertiary settings, and involves participation by a variety of practitioners, the Clinical Senate membership must reflect this diversity. Although clinicians are nominated by service providers, it is important for each Senator to recognise they do not represent the nominating body. There is an expectation that clinicians will take a whole-of-system perspective, partner with consumers and health managers, and be inclusive and multidisciplinary in approach.

\section{Rural}

Given the size of Western Australia and the service delivery challenges faced by WA Health, the Senate's Terms of Reference specify that $25 \%$ of its senators, as a minimum, should be rural clinicians who can give voice to the issues facing this population (particularly indigenous groups) and those providing health care services to rural and remote communities. The contribution from Aboriginal Health Workers during Senate debates is invaluable.

\section{Mission and values}

Senators must be willing to abide by the core mission and values outlined in the Clinical Senate of Western Australia Terms of Reference [9].

These include:

- Independence that strives to put aside personal allegiances and political agendas

- Clinical leadership with integrity.

- Informed debate, led by current evidence.

- Respect for diversity of opinions and promotes and practices equity.

- Issues considered are timely and responsive to the needs of the whole of Health System.

The Queensland Clinical Senate has adopted many similar values for their membership. Their terms of reference state:

"There has been an expectation that clinicians will take a wholeof-system perspective, partner consumers and managers, and be multidisciplinary in nature" [15].

\section{Individual power to influence change}

In considering how the WA Clinical Senate engages clinicians to focus on transformational change of the health care system, it is useful to consider literature in the areas of clinical engagement and influence strategies in healthcare organisations.

In the article by Sephar et al., the authors state that resistance to change in healthcare organisations can be linked to power battles within the structure of the organisation [16]. Day to day decision-making is 
influenced more by staff on the front-line rather than by those with more formal power, who are higher up the organisational chain.

Sephar et al. undertook a study into the influence strategies used by nursing and medical staff in Norwegian hospitals using "profession neutral" management [16]. The authors reviewed various literatures that applied sociological theory to organisational change and also to clinical engagement, stating that policy makers hoping for system wide change need to have an understanding of professional power bases and influence strategies existing in healthcare organisations which can result in resistance to change.

Power in decision making in healthcare falls into different categories and types. One common classification distinguishes between the power an individual holds in relation to their status or position and embodies notions of reward, legitimate or coercive power (position power) versus power associated with referent or perceived attractiveness and/or expert power (personal power) [16,17].

Sephar et al. analysed decision making and power structures in health and concluded that within many hospitals "power lies in expertise and that managers with a clinical background are more likely to draw on expert power than on formal position power" [16].

They concluded that a manager's professional or clinical background could serve as both a resource for influencing upwards or downwards decision-making, as well as a constraint. The Clinical Senate aims to engage a range of representation from across the whole of the WA health system thus hoping to minimise the influence of any power bases from a particular sector, profession, management, or group. As outlined previously, Senators are nominated and selected according to their ability to consider what is in the best interest for the whole healthcare system in Western Australia. When a proxy position becomes available, nominations are considered in relation to the added value their relevant expertise on the topic will add to make the depth and breadth of discussions, and whether the proxy will fulfil the duties expected of the senate membership in establishing whole-of-system recommendations rather than attending with a personal or organisational agenda.

The Senate terms of reference state that people are to "vote on their individual opinion, rather than to represent others or their organisation" facilitating a process free of power enclaves, so that each individual can form and freely express their own opinion and vote on recommendations without coercion, punishment or retribution stemming from any individual or organisational power imbalances [9].

\section{The Role of the Clinical Senate}

\section{The clinician voice}

The core role of the Clinical Senate is to debate topics of significant importance to the state and clinicians in order to develop recommendations for the Director General and Minister of Health that will improve health service delivery in the interests of the health of all West Australians. Debates commence with a broad overview of the topic's key issues from different angles or perspectives rather than a biased or narrow view. The facilitated plenary debate affords the opportunity for individuals to speak freely.

Thus, an individual's power may be exercised through the Senate's various platforms. This may be in the form of the plenary debate, asking or responding to specific questions adding depth to the topic, discussion within the small workshop groups, and ultimately voting on the debate recommendations, which utilise a fair and equitable consensus approach across the senate floor.

\section{The decision-making process}

The decision-making model of the WA Clinical Senate has evolved over time. First described by Blackwell et al in 2009, the senate process utilizes the Deliberative Decision Making Model (Figure 1) where a diverse group of people are brought together for a common purpose [18]. The model has been applied in a wide variety of contexts, such as Constitutional Conventions and Citizens' Juries (Figure 1). This model is an effective method of sharing diverse perspectives and working towards a consensus outcome that ensures shared ownership of the results.

A typical WA Clinical Senate debate is a full day session that commences with a series of presentations from experts in the field carefully curated to provide a balance of information to inform the debate. A consumer perspective is an important component of the evidence brought to the table. Pre-reading sent to senators in advance of the meeting supports this information.

The Executive Sponsor for each debate is carefully chosen as a senior Health figure who is well informed on the subject at hand and well placed to advocate for, and influence the implementation of, recommendations from the debate. They contribute to the first session by setting the scene, offering probing questions and outlining their expectations of the outcomes.

A full plenary session affords all senators the opportunity to contribute perspectives drawn from their unique clinical experience and expertise. A skilled facilitator ensures a robust debate with balanced sharing of the diversity of views. Expert witnesses, invited for either their experience or expertise on the matters at hand, add to the wisdom in the room and advise and guide the discussions.

The afternoon is devoted to workshops for the forming of recommendations, an activity limited to senators and the Executive Sponsor such that the outcomes are not driven by or become a wish list of, the experts in the field, but rather are the results of the due deliberations of the experienced, informed clinicians. The final session of the day engages the full Senate chamber in reviewing and prioritising the recommendations formed in the workshops such that all senators have ownership of the final set of up to 10 recommendations.

These recommendations are reported as a raw product. They may be edited for clarity, so that time in the workshops is not wasted on

\section{Deliberative Decision Making Model Key Features}

- Broad cross-sectional membership

- Commitment to making decisions in the best interests of the whole community

- Provision and consideration of unbiased information and evidence on the given subject

- Time to deliberate

- Decisions are required

- A guarantee that the work will be heard and acted upon

Figure 1: Key features of the deliberate decision-making model $[17,18]$ 
minor word-smithing, but the essential intent is carried over into the final report. The recommendations are not worked up by any external or policy group ensuring that they remain truly representative of the clinical voice when received by the Director General (DG) of the day and their team.

\section{Effectiveness in facilitating clinician engagement}

There is evidence that clinicians value the opportunity to contribute to state-wide health reform. In a 2016 survey, $82 \%$ of responders agreed that the Senate provides a mechanism for them to give feedback to the key decision makers in Health. $87 \%$ agreed that it gave them an opportunity to work on recommendations that could make a difference to the health of West Australians and $88 \%$ agreed that it allowed them to consider and contribute to bigger picture health reform.

\section{Implementation and Accountability of Recommenda- tions}

Blackwell et al. in 2009 highlighted the lack of a mandate to enforce recommendations as a weakness of a Clinical Senate [18]. At that time, the Executive Sponsor was required to report on progress of the recommendations a year after the DG and the Executive Forum had received them.

Although this provided a level of accountability for implementation it did not guarantee progress. The process was enhanced considerably in early 2010 when Kim Snowball, the DG at the time, agreed to endorse the recommendations he felt should be implemented.

The level of endorsement (i.e., Endorsed, Endorsed in Principle, Not Endorsed), is reported directly back to the Senate members by the DG or his delegate at the subsequent debate, providing immediate feedback to senators on the outcomes and demonstrating the value of their work. The Senate thereby delivers on the last key characteristic identified in the Deliberative Decisions Making Model: "A guarantee that the work will be heard and acted upon".

The success of this level of accountability and method of reporting was born out by an audit of the implementation of endorsed recommendations that found that $82 \%$ were at least partially implemented with $45 \%$ fully or substantially implemented. The study also demonstrated that recommendations endorsed by the DG were more than twice as likely to be fully or substantially implemented (54\%) than those endorsed in principle (20\%) [19,20].

In 2016, a new challenge to the accountability for getting traction on recommendations faces the Clinical Senate with the implementation of governance reforms in WA Health. It will be critical that under the proposed changes clear pathways for endorsement of recommendations and accountability for implementation are sustained.

The Health Services Bill 2016 provides a devolved model of governance with decision-making closer to service delivery and patient care [20]. It is at those key points that Senate recommendations will have the greatest impact if they are to benefit the health of all West Australians.

Under the new bill, Health Services will become separate statutory authorities with their Boards legally responsible and accountable for the delivery of health services in their local communities [21]. Thus it would be appropriate for Boards to require their Executive to report to them on the progress of Senate recommendations in their Health Service.
The Director General in leading the Department of Health, reformed as the System Manager, would remain the appropriate point of oversight of the Clinical Senate as the key state-wide clinician engagement mechanism, continuing to receive and endorse recommendations. Reporting by the Health Services on the progress of endorsed recommendations to this central point would allow consistent ongoing measurement of the extent of implementation and success of the reforms across WA Health.

The governance reforms will also see an opportunity to increase clinician engagement in WA at the local health service level. Like their predecessors, the Governing Councils, the Boards will have a specific mandate for consulting with their clinicians regarding the provision of health services [21]. This presents an opportunity for local clinician engagement bodies or mechanisms established to be linked to the Clinical Senate to leverage the work at both statewide and local levels and avoid duplication. Leverage such as this has been achieved to good effect through linkage of the Clinical Senate and the Health Networks, another effective and important clinician engagement mechanism over the last decade in WA, where combined effort has provided a broader reach and stronger outcomes on debates such as End of Life Care, Women's Health, Youth Health and Aged Care.

\section{Summary}

Clinical engagement is important to improve quality and safety, effectiveness and efficiency of health care organisations [1-3]. The key elements of a state-wide clinical engagement body are culture and leadership, membership, mission and values, decision-making processes and debate to generate recommendations. Finally there is a need for accountability for implementation of recommendations. The WA Clinical Senate is a model that currently addresses all these main components and is available to government to achieve state-wide clinical engagement.

\section{References}

1. The King's Fund (2012) Leadership and engagement for improvement in the NHS, Together we can. Report from The King's Fund Leadership Review.

2. Alimo-Metcalf B, Alban-Metcalf J (2008) Engaging leadership: Creating organisations that maximise the potential of people. London: Chartered Institute of Personnel and Development.

3. Clement L, Soroka S, Robblee J (2012) Increasing physician engagement Quality in Healthcare 4: 22.

4. Griffiths Report on NHS (1983) Department of Health and Social Security.

5. Report of the Mid Staffordshire NHS Foundation Trust Inquiry (2013) Chaired by Robert Francis QC.

6. Business Dictionary (2016) Organizational Culture, Definition

7. Davies R, Dugas J, Cox C (2013) The role of the board and the practice of directorship. In Australian Institute of Company Directors, Company Directors Course 1-78.

8. Quinlivan JA, Miller M, Hutton M (2016) Does a Clinical Senate enhance state-wide clinician engagement? A survey study (In press). Australian Health Review.

9. Department of Health, Western Australia (2016) The Clinical Senate of Western Australia, Terms of Reference.

10. Department of Health, Western Australia (2015) The Clinical Senate of Western Australian Charter.

11. Managing Groups and Teams/Diversity (2016) Wikibooks.

12. Gibson K (2012) Excellence in clinical engagement. Medical Forum WA 27.

13. Vroom VH (1964) Work and motivation. Wiley Carnegie Work and motivation Pittsburgh, PAJ, New York 331.

14. May J (2015) Clinical service integration: A stocktake of the Australian experience. Future Hospital Journal 2: 142-146. 
Citation: Quinlivan JA, Basile T, Gibson K, Xu D, Croker N (2016) The Western Australian Clinical Senate as a Model for State-Wide Clinical Engagement. Health Care: Current Reviews 4: 172. doi: 10.4172/2375-4273.1000172

15. Queensland Health (2015) Queensland Government, Queensland Clinical Senate, Terms of Reference.

16. Sephar I, Frich J, Kjekshus E (2014) Clinicians in management: A qualitative study of managers' use of influence strategies in hospitals. BMC Health Service Research 14: 251.

17. Northouse PG (2012) Leadership: Theory and practice. Thousand Oaks, CA: Sage.

18. Blackwell (2009) Great debate: How clinicians make their views heard in health reform. Australian Health Review 33: 5-10.
19. Mclver S (1998) Healthy debate: An independent evaluation of citizen's juries in health settings. Kings Fund, London.

20. Department of Health (2014)WesternAustralia. Review of the Recommendations of the Clinical Senate of Western Australia Debates. Perth: Health Strategy and Networks Branch, Department of Health, Western Australia.

21. Department of Health (2016) Role of health services and boards: Strong governance foundations for a safe, high quality, sustainable WA Health System. Health Reform Fact Sheet. 